\title{
Parameter Influence on the Harmonically Excited Duffing Oscillator
}

\author{
Livija Cveticanin $^{1}$, Gyula Mester $^{2}$ and Istvan Biro ${ }^{2}$ \\ ${ }^{1}$ Faculty of Technical Sciences, Trg D. Obradovica 6, 21000 Novi Sad, Serbia \\ cveticanin@uns.ac.rs \\ ${ }^{2}$ University of Szeged, Faculty of Engineering, Mars tér 7, H-6724 Szeged, \\ Hungary; gmester@inf.u-szeged.hu; biro-i@mk.u-szeged.hu
}

\begin{abstract}
In this paper the influence of the initial conditions and the interaction of the parameters on the motion of the strong nonlinear Duffing oscillator are investigated. The initial conditions are arbitrary and need not be zero. An analytical procedure for solving the strong nonlinear differential equation with excitation term is developed. The obtained solutions give the physical explanation of the excited vibrations caused by the excitation force and non-zero initial conditions. The analytical results are compared with numerical results and show good agreement.
\end{abstract}

Keywords: Duffing oscillator; harmonic excitation; non-zero initial conditions; jump effect

\section{Introduction}

Vibration data, obtained by repeated measurement in a forced system (for example, motor-support system), represent a spectra of different values, in spite of the fact, that the measuring position and conditions, as well as, the excitation of the system has not changed. Using the theoretical consideration of the excited vibration of non-linear systems, it is to be expected that the obtained results have to be highly repeatable and to satisfy certain rules (see [1]-[13] and References mentioned in them). For all of the aforementioned analytical investigations, it is common to assume that the motion is steady state, with non-zero initial conditions. Namely, it is stated that the initial conditions have nothing to do with the long term motion properties and need not to be taken into account. The discrepancy between the experimental and analytical values gives us an idea to investigate nonlinear excited vibrations by including the initial conditions. In the paper [14], the vibrations of a harmonically excited pure nonlinear oscillator (the linear displacement term fails) with non-zero initial conditions is analyzed. It has been noticed that in spite of long term motion, the influence on the initial conditions on the motion remains. The intention of this paper is to analyze the 
effect of the initial conditions on the motion of an excited nonlinear oscillator of Duffing type. For this model the linear displacement force is also taken into consideration. The model of the system is a strong nonlinear second order differential equation with an excitation term

$\ddot{y}+\alpha y+\gamma y^{3}=F_{0} \cos (\Omega t)$,

and arbitrary initial conditions

$y(0)=y_{0}, \quad \dot{y}(0)=\dot{y}_{0}$,

where $\alpha$ and $\gamma$ are the coefficients of the linear and cubic terms, $F_{0}$ and $\Omega$ are the excitation amplitude and frequency, $y_{0}$ and $\dot{y}_{0}$ are the initial displacement and velocity, respectively. The solution of (1) is assumed in the form which is usual for the linear vibration model. Using the harmonic balance method the coefficients of the solution are determined. For the special group of parameter values the jump-up and jump-down phenomena are investigated with special attention to the influence of the initial values. The interaction between the oscillator parameters and arbitrary initial conditions on the vibration are also analyzed. For certain numerical values the analytically obtained results are compared with numerical results. They are in good agreement.

\section{Analytical Solving Procedure}

Let us assume the solution of (1) as a sum of trigonometric functions

$$
y=A \cos (\Omega t)+B \cos (\omega t)+C \sin \left(\omega_{1} t\right),
$$

where $A, B, C$ and also $\omega$ and $\omega_{1}$ are unknown values. This form corresponds to the usual solution of the harmonically excited linear oscillator. Substituting (3) and its first and second time derivative into (1) we obtain

$$
\begin{aligned}
& F_{0} \cos (\Omega t)=\left(\alpha-\Omega^{2}\right) A \cos (\Omega t)+\left(\alpha-\omega^{2}\right) B \cos (\omega t)+\left(\alpha-\omega_{1}^{2}\right) C \sin \left(\omega_{1} t\right) \\
& +\gamma\left(A \cos (\Omega t)+B \cos (\omega t)+C \sin \left(\omega_{1} t\right)\right)^{3} .
\end{aligned}
$$

Separating the terms with the first order trigonometric functions $\cos (\Omega t), \cos (\omega t)$ and $\sin \left(\omega_{1} t\right)$ in (4), the following system of three algebraic equations is obtained

$$
\begin{aligned}
& \cos (\Omega \mathrm{t}): \quad\left(\alpha-\Omega^{2}\right) A+\frac{3}{4} \gamma A\left(A^{2}+2 B^{2}+2 C^{2}\right)=F_{0}, \\
& \cos (\omega t): \quad\left(\alpha-\omega^{2}\right)+\frac{3}{4} \gamma\left(2 A^{2}+B^{2}+2 C^{2}\right)=0, \\
& \sin \left(\omega_{1} t\right): \quad\left(\alpha-\omega_{1}^{2}\right)+\frac{3}{4} \gamma\left(2 A^{2}+2 B^{2}+C^{2}\right)=0 .
\end{aligned}
$$


The initial conditions (2) with (3) give two additional algebraic equations

$B=y_{0}-A, \quad \omega_{1}=\dot{y}_{0} / C$.

Substituting (6) into (5) $)_{3}$ the parameter $C$ as the function of $A$ follows

$$
C^{2}=\frac{2}{3 \gamma}\left[\alpha+\frac{3 \gamma\left(A^{2}+\left(y_{0}-A\right)^{2}\right)}{2}\right]\left[\sqrt{1+\frac{3 \dot{y}_{0}^{2}}{\left[\alpha+3 \gamma\left(A^{2}+\left(y_{0}-A\right)^{2}\right) / 2\right]^{2}}}-1\right] .
$$

Using (6) and (7), the relations $(5)_{1}$ and $(5)_{2}$ transform into

$$
\begin{aligned}
& F_{0}=\left(\alpha-\Omega^{2}\right) A+\frac{3 \gamma A}{4}\left(A^{2}+2\left(y_{0}-A\right)^{2}\right) \\
& +\frac{4 A}{3 \gamma}\left(\sqrt{\left(\alpha+\frac{3}{2}\left(A^{2}+\left(y_{0}-A\right)^{2}\right)\right)^{2}+3 \dot{y}_{0}^{2}}-\left(\alpha+\frac{3}{2}\left(A^{2}+\left(y_{0}-A\right)^{2}\right)\right),\right.
\end{aligned}
$$

and

$$
\begin{aligned}
& 0=\left(\alpha-\omega^{2}\right)+\frac{3 \gamma}{4}\left(2 A^{2}+\left(y_{0}-A\right)^{2}\right) \\
& +\frac{4}{3 \gamma}\left(\sqrt{\left(\alpha+\frac{3}{2}\left(A^{2}+\left(y_{0}-A\right)^{2}\right)\right)^{2}+3 \dot{y}_{0}^{2}}-\left(\alpha+\frac{3}{2}\left(A^{2}+\left(y_{0}-A\right)^{2}\right)\right)\right) .
\end{aligned}
$$

Eq. (8) is a sixth order algebraic equation for parameter $A$. The solution for $A$ has to be substituted into (9) and the solution for $\omega$.

\section{Solution for Non-Zero Initial Displacement}

For the case when the initial velocity is zero $\left(\dot{y}_{0}=0\right)$ but the initial displacement is a non-zero one $\left(y_{0} \neq 0\right)$

$$
y(0)=y_{0}, \quad \dot{y}(0)=0,
$$

the aforementioned relations (7)-(9) transform into

$$
\begin{aligned}
& \left(\alpha-\Omega^{2}\right) A+\frac{3}{4} \gamma A\left(A^{2}+2 B^{2}\right)=F_{0}, \quad\left(\alpha-\omega^{2}\right)+\frac{3}{4} \gamma\left(2 A^{2}+B^{2}\right)=0, \\
& C=0, \quad \omega_{1}=0, \quad B=y_{0}-A .
\end{aligned}
$$

The general solution (3) simplifies into

$y=A \cos (\Omega t)+\left(y_{0}-A\right) \cos (\omega t)$, 
where $A$ and $\omega$ are the solutions of the equations

$$
\begin{aligned}
& \frac{9}{4} \gamma A^{3}-3 y_{0} \gamma A^{2}+\left(\alpha-\Omega^{2}+\frac{3}{2} \gamma y_{0}^{2}\right) A-F_{0}=0, \\
& \omega^{2}=\alpha+\frac{3}{2} \gamma A^{2}+\frac{3}{4} \gamma\left(y_{0}-A\right)^{2} .
\end{aligned}
$$

The relation $(13)_{2}$ can be rewritten in the form

$\omega^{2}=\omega_{f}^{2}-\frac{3}{2} \gamma A\left(y_{0}-\frac{3}{2} A\right)$

where $\omega_{f}=\sqrt{\alpha+3 \gamma y_{0}^{2} / 4}$ is the approximate frequency of free vibration [15]. Eq. (14) represents the corrected frequency of vibration caused by harmonic excitation. The frequency depends not only on the initial amplitude, as in the case for free vibrations of cubic nonlinear oscillator, but also on the excitation properties. Introducing the new variable $A=Y+4 y_{0} / 9$ into $(13)_{1}$, we have a cubic algebraic equation.

$Y^{3}+p Y+q=0$

where

$$
p=\frac{12\left(\alpha-\Omega^{2}\right)+2 \gamma y_{0}^{2}}{27 \gamma}, \quad q=Q-\frac{4 F_{0}}{9 \gamma}, \quad Q=\frac{144 y_{0}\left(\alpha-\Omega^{2}+88 \gamma y_{0}^{3}\right.}{729 \gamma} .
$$

The number of real solutions of (15) depends on the relation between the parameters of the excitation force $\mathrm{F}_{0}$ and $\Omega$, ant the initial displacement $y_{0}$. As suggested by mathematicians the number of real solutions (one or three) depends on the sign of the discriminant $D=\left(q^{2} / 4\right)+\left(p^{3} / 27\right)$ which is according to (16)

$$
D=\frac{1}{4}\left(Q-\frac{4 F_{0}}{9 \gamma}\right)^{2}+\frac{p^{3}}{27}
$$

For $D>0$ the number of real solutions of (15) is one and for $D<0$ it is three. The boundary discriminant of $(13)_{1}$ is $D=0$, when two of the real solutions are equal and the third differs.

\subsection{Discussion of the Domain of Solutions}

For computational reasons, let us rewrite the relation (17) in the form more suitable for analysis

$$
D=\left(\frac{2}{9 \gamma}\right)^{2}\left(F_{0}-\frac{9 \gamma Q}{4}-\frac{9 \gamma}{2} \sqrt{\frac{|p|^{3}}{27}}\right)\left(F_{0}-\frac{9 \gamma Q}{4}+\frac{9 \gamma}{2} \sqrt{\frac{|p|^{3}}{27}}\right),
$$


where according to (16) the $\Omega(p)$ and $Q(p)$ functions are

$$
\Omega=\sqrt{\alpha-\frac{1}{2}\left(27 \gamma p-2 \gamma y_{0}^{2}\right)}, \quad Q=\frac{4 p y_{0}}{9}+\frac{64 y_{0}^{3}}{729} .
$$

The discriminant (18) is zero for (19) and

$$
F_{01}=\frac{16 \gamma}{81} y_{0}^{3}+\gamma p y_{0}-\frac{\gamma|p|}{2} \sqrt{3|p|}, \quad F_{01}=\frac{16 \gamma}{81} y_{0}^{3}+\gamma p y_{0}+\frac{\gamma|p|}{2} \sqrt{3|p|} .
$$

Substituting(19) into (20) the boundary $F_{0}-\Omega$ functions for various values of initial displacement $y_{0}$ are obtained as

$$
\begin{aligned}
& F_{01}=\frac{16 \gamma y_{0}^{3}}{81}+\frac{\left|12\left(\alpha-\Omega^{2}\right)+2 \gamma y_{0}^{2}\right|}{27}\left(y_{0}-\frac{1}{2} \sqrt{\left|\frac{\left|12\left(\alpha-\Omega^{2}\right)+2 \gamma y_{0}^{2}\right|}{9 \gamma}\right|}\right), \\
& F_{01}=\frac{16 \gamma y_{0}^{3}}{81}+\frac{\left|12\left(\alpha-\Omega^{2}\right)+2 \gamma y_{0}^{2}\right|}{27}\left(y_{0}+\frac{1}{2} \sqrt{\left|\frac{\left|12\left(\alpha-\Omega^{2}\right)+2 \gamma y_{0}^{2}\right|}{9 \gamma}\right|}\right)
\end{aligned}
$$

Analyzing the relations (19) and (21) it is obvious that for

$$
p=\frac{16 y_{0}^{2}}{27}, \text { i.e., } \Omega=\sqrt{\alpha+\frac{3}{2} \gamma_{0}^{2}},
$$

the excitation amplitude is minimal and has the value $F_{0 \min }=0$.

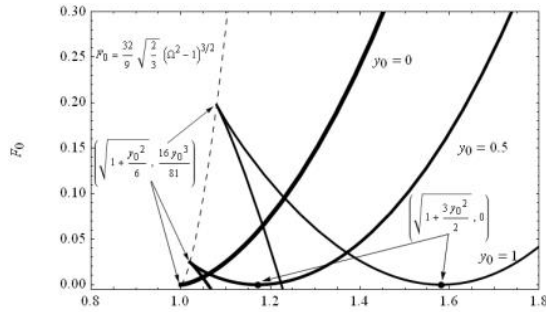

$\Omega$

Figure 1

$F_{0}-\Omega$ curves with characteristic points for $\alpha=\gamma=1$ and various initial displacements

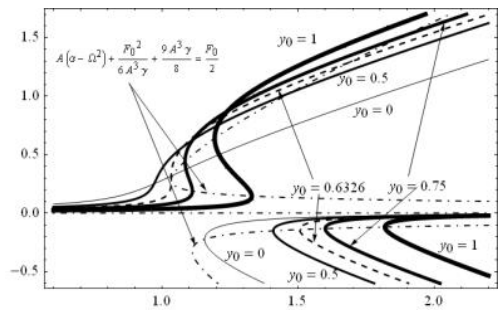

Figure 2

$A-\Omega$ curves with characteristic points $\alpha=\gamma=1$, $F_{0}=0.05$ and various initial displacements

In addition, if the discriminant is zero, it follows

$\Omega=\sqrt{\alpha+\frac{\gamma y_{0}^{2}}{6}}, \quad F_{0}=\frac{16 \gamma y_{0}^{3}}{81}$,

as the value of $p$ is zero. The values (22) and (23) correspond to two characteristic points in the $F_{0}-\Omega$ plane. In Fig. 1 the $F_{0}-\Omega$ curves (21) for several values of initial 
displacements $\left(y_{0}=0 ; 0.5 ; 1\right)$ are plotted. The parameters of the system are $\alpha=1$ and $\gamma=1$. The excitation amplitude is positive, i.e., $F_{0}>0$. It can be seen that for $y_{0}=0$ the $F_{0}-\Omega$ curve is single-valued. Namely, the relations (21) transform into only one and give a curve which separates the $F_{0}-\Omega$ plane into two regions: left from the limit curve where the discriminant $D$ is positive and right of the curve where the discriminant is not positive $(D \geq 0)$. For $y_{0}>0$, the $F_{0}-\Omega$ curves are multi-valued. The two curves (21) give in the $F_{0}-\Omega$ plane an additional region where $D \geq 0$. The obtained region is larger for higher values of initial displacement. The $\Omega$ and $F_{0}$ coordinates for the peak points of the region are $\left(\sqrt{1+y_{0}^{2} / 6}, 16 y_{0}^{3} / 81\right)$ and $\left(\sqrt{1+3 y_{0}^{2} / 2}, 0\right)$ according to (22) and (23). The higher the value of $y_{0}$ the peak values move toward higher values of $\Omega$ and $F_{0}$. For $F_{0}$ higher than the peak value only one jump frequency is evident for which the discriminant changes the sign. For the 'jump frequency' there is the transition from the region where $D>0$ to the region where $D<0$.

\subsection{Analytical Expression of Solutions}

As it was previously mentioned the number of real solutions of $(13)_{1}$ depends on the sign of the discriminant $D$.

a) For $\mathrm{D}=0$ three real solutions of $(13)_{1}$ exist, where two of them are equal

$$
A_{1}=\frac{4 y_{0}}{9}+2 \sqrt[3]{-\frac{Q}{2}+\frac{2 F_{0}}{9 \gamma}}, \quad A_{2,3}=\frac{4 y_{0}}{9}+\sqrt[3]{\frac{Q}{2}-\frac{2 F_{0}}{9 \gamma}} .
$$

b) For the values of excitation $F_{0}$ and $\Omega$ which are inside the area (21) the discriminant is negative and three real solutions are

$$
A_{1}=\frac{4 y_{0}}{9}+K \cos \frac{\varphi}{3}, \quad A_{2}=\frac{4 y_{0}}{9}+K \cos \frac{\varphi+2 \pi}{3}, \quad A_{3}=\frac{4 y_{0}}{9}+K \cos \frac{\varphi+\pi}{3},
$$

where

$$
K=2 \sqrt{-\frac{p}{3}}, \quad \cos \varphi=-\frac{Q}{2}+\frac{2 F_{0}}{9 \gamma}\left(-\frac{p}{3}\right)^{-3 / 2}
$$

c) Outside the region (21), where the discriminant $D$ of $(13)_{1}$ is positive, only one real solution exists

$$
A=\frac{4 y_{0}}{9}+\sqrt[3]{-\frac{Q}{2}+\frac{2 F_{0}}{9 \gamma}+\sqrt{D}}+\sqrt[3]{-\frac{Q}{2}+\frac{2 F_{0}}{9 \gamma}-\sqrt{D}}
$$


The result (27) is valid for all values of $F_{0}$ and for the excitation frequency which satisfies the relation

$\Omega<\sqrt{\alpha+\frac{1}{6} \gamma y_{0}^{2}}$

where the parameter $p$ and the discriminant are always positive.

In Fig. 2 according to (23)-(27) the response amplitude A as a function of the excitation frequency $\Omega$ for several initial displacements $\left(y_{0}=0 ; 0.5 ; 0.6326 ; 0.75 ; 1\right)$ is shown. The excitation amplitude is $F_{0}=0.05$ and the parameters of the oscillator are $\alpha=\gamma=1$. We note that, depending on the value of $y_{0}$, some of the curves are multi-valued while others are single-valued. It is of special interest to determine the characteristic points in $A-\Omega$ curves where the bifurcation of the solution appears.

\subsection{Characteristic Points}

Let us rewrite the relation $(13)_{1}$ into the form

$$
\Omega=\sqrt{\frac{9 \gamma A^{2}}{4}-3 y_{0} \gamma A-\frac{F_{0}}{A}+\frac{3 \gamma y_{0}^{2}}{2}+\alpha}
$$

To obtain the characteristic peak values in the aformentioned $A-\Omega$ curves, the first derivative of (29) for $\Omega$ as a function of the amplitude $A$ has to be equated with zero

$$
\frac{9}{2} \gamma A^{3}-3 y_{0} \gamma A^{2}+F_{0}=0
$$

The cubic algebraic equation has one or three real solutions dependent on the sign of the discriminant

$$
D_{1}=\frac{F_{0}}{81 \gamma^{2}}\left(F_{0}-\frac{16 \gamma y_{0}^{3}}{81}\right)
$$

For $D_{1}>0$, when $F_{0}>16 \gamma y_{0}^{3} / 81$, only one real solution for $A$ exists

$$
A_{m}=\frac{2 y_{0}}{9}+\sqrt[3]{\frac{1}{9}\left(-\frac{F_{0}}{\gamma}+\frac{8 y_{0}^{3}}{81}\right)+\sqrt{D_{1}}}+\sqrt[3]{\frac{1}{9}\left(-\frac{F_{0}}{\gamma}+\frac{8 y_{0}^{3}}{81}\right)-\sqrt{D_{1}}}
$$

For $D_{1}=0$, i.e., $F_{0}=16 \gamma y_{0}^{3} / 81$ two real solutions exist 


$$
A_{m 1}=-\frac{2 y_{0}}{9}, \quad A_{m 2, m 3}=\frac{4 y_{0}}{9}
$$

For $D_{1}<0$, i.e., $0<F_{0}<16 \gamma y_{0}^{3} / 81$ three real solutions exist

$$
\begin{aligned}
& A_{m 1}=\frac{2 y_{0}}{9}\left(1+2 \cos \frac{\psi}{3}\right), \quad A_{m 2}=\frac{2 y_{0}}{9}\left(1+2 \cos \frac{\psi+2 \pi}{3}\right) \\
& A_{m 2}=\frac{2 y_{0}}{9}\left(1+2 \cos \frac{\psi+4 \pi}{3}\right)
\end{aligned}
$$

where

$$
\cos \psi=1-\frac{81 F_{0}}{8 y_{0}^{3} \gamma}
$$

Analyzing the relation (30) the initial displacement as the function of the response and excitation amplitude is expressed

$$
\frac{1}{3 \gamma A^{2}}\left(\frac{9}{2} \gamma A^{3}+F_{0}\right)=y_{0}
$$

Substituting (36) into (13) $)_{1}$ we eliminate the initial displacement and we have the $A(\Omega)$ function for various values of $F_{0}$

$$
\left(\alpha-\Omega^{2}\right) A+\frac{9}{8} \gamma A^{3}-\frac{F_{0}}{2}+\frac{F_{0}^{2}}{6 \gamma A^{3}}=0
$$

Substituting the values of the amplitudes $A_{m}$ (32)-(34) into (37) the frequencies $\Omega_{m}$ are obtained

$$
\Omega_{m i}=\sqrt{\alpha+\frac{9}{8} \gamma A_{m i}^{2}-\frac{F_{0}}{2 A_{m i}}+\frac{F_{0}^{2}}{6 \gamma A_{m i}^{4}}}
$$

where $i=1,2,3$. The values $\left(A_{m}, \Omega_{m}\right)$ correspond to certain constant value of $F_{0}$ and initial displacement $y_{0}$.

In Table 1, the characteristic amplitudes and frequencies for the oscillator with parameter values $F_{0}=0.05, \alpha=\gamma=1$ and various initial conditions $y_{0}$ are calculated.

It can be concluded that for $y_{0}<0.6326$ one solution exists, while for $y_{0} \geq 0.6326$ number of solutions is three. Comparing the values in Table 1 with the characteristic points in Fig. 2, it can be seen that they are in good agreement. 
Table 1

Characteristic amplitudes and frequencies for various initial conditions

\begin{tabular}{|c|c|c|c|c|c|c|}
\hline$Y_{0}$ & $A_{1}$ & $\Omega_{1}$ & $A_{2}$ & $\Omega_{2}$ & $A_{3}$ & $\Omega_{3}$ \\
\hline 0 & -0.22314 & 1.1559 & & & & \\
\hline 0.5 & -0.15140 & 1.4085 & & & & \\
\hline 0.63257 & -0.14057 & 1.5057 & 0.28114 & 1.0328 & 0.28114 & 1.0328 \\
\hline 0.75 & -0.13254 & 1.5996 & 0.18902 & 1.1110 & 0.44351 & 1.0843 \\
\hline 1 & -0.11893 & 1.8191 & 0.14610 & 1.3295 & 0.63950 & 1.1931 \\
\hline
\end{tabular}

\subsection{Explanation of the Solution}

Based on the approximate analytical solution (12), and results given in the previous Section, the physical phenomena and vibration of the harmonically excited Duffing oscillator with non-zero initial displacement are discussed. The parameters $\alpha$ and $\gamma$ and also the initial displacement $y_{0}$ are treated as constant values. For the constant values of excitation amplitude $F_{0}$ the excitation frequency $\Omega$ is increased from zero to infinity.

1) For the excitation frequency $\Omega \approx \varepsilon>0$, where $\varepsilon$ is a small positive value, the parameter $A$ is positive and satisfies the relation $A<y_{0}$ (see Eq. (13) $)_{1}$. The approximate solution (12) for vibration simplifies into

$y \approx A+\left(y_{0}-A\right) \cos (\omega t)$

where

$\omega \approx \omega_{f} \sqrt{1-\frac{3 \gamma A y_{0}}{2 \omega_{f}^{2}}}$

The harmonic vibration with amplitude (y0-A) and frequency $\omega(40)$ is around the position $A$. If the calculated parameter $A$ is small, i.e. $A<<y_{0}$, the relation (39) simplifies into

$y=y_{0} \cos (\omega t)$

The frequency of excited vibration $\omega$ is smaller than the frequency of free vibration $\omega_{f}$. The period of excited vibration is longer than for free vibration of the system. Comparing the excited frequency $\omega$ with the excitation frequency $\Omega$ it has been concluded that the second is significantly smaller than the first one.

2) For the excitation frequency

$$
\Omega^{*}=\sqrt{\left(\alpha+\frac{3}{4} \gamma y_{0}^{2}\right)-\frac{F_{0}}{y_{0}}}=\omega_{f} \sqrt{1-\frac{F_{0}}{y_{0} \omega_{f}^{2}}}
$$


we have $A=y 0$. The solution (12) simplifies into

$y=y_{0} \cos \left(\Omega^{*} t\right)$

The vibration is harmonic in nature, with amplitude $y_{0}$ and frequency $\Omega^{*}$ which represents the corrected value of the frequency of free vibration of the system $\omega_{f}$. The correction parameter depends on the excitation amplitude and the initial displacement. The higher the amplitude of excitation, the more significant is the variation of the frequency to that of free vibration. The period of excited vibration is longer than for free vibration. The relation (42) is valid only for $F_{0} /\left(y_{0} \omega_{f}^{2}\right) \leq 1$.

3) For $\Omega>\Omega_{\mathrm{m} 1}$, where $\Omega_{\mathrm{m} 1}$ is the jump down frequency (37) in $A-\Omega$ diagram (see Table 1), the calculated parameter $A$ becomes negative with absolute value $|A|<<y_{0}$. The oscillations are approximately periodical (39) with frequency

$\omega \approx \omega_{f} \sqrt{1+\frac{3 \gamma|A| y_{0}}{2 \omega_{f}^{2}}}$

Due to (44) it is evident that the frequency of excited vibration $\omega$ is higher than for free vibration $\omega_{f}$ and the period of excited vibration is shorter than for free vibration. For $\Omega>>\omega_{f}$ the value $|A|$ tends to zero and the frequency of vibration is close to $\omega_{f}$. The effect of excitation disappears.

4) According to (13) $)_{1}$ it is evident that for $\Omega<\Omega^{*}$ the parameter $A$ satisfies the relation $A<y 0$. As the difference between the excited and excitation frequency is a small positive value $\varepsilon$, i.e. $\omega-\Omega=\varepsilon>0$, it is convenient to rewrite the solution (12) into the form

$y=y_{0} \cos \Omega t-2\left(y_{0}-A\right) \sin \left(\frac{\omega-\Omega}{2} t\right) \sin \left(\frac{\omega+\Omega}{2} t\right)$

which is suitable for analysis. The second term in the relation (45) describes trembling with negative amplitude and period $T=2 \pi /(\omega-\Omega)$. The period of trembling is lengthened by decreasing the difference between the frequencies $\omega$ and $\Omega$. The period is longest for the minimal value of the difference $(\omega-\Omega)$.

5) For $\Omega^{*}<\Omega<\Omega_{\mathrm{m} 1}$ the parameter $A$ satisfies the relation $A>y_{0}$ and according to (13) the difference between the excited and excitation frequencies is positive. The relation transforms into

$$
y=y_{0} \cos (\Omega t)+2\left(A-y_{0}\right) \sin \left(\frac{\omega-\Omega}{2} t\right) \sin \left(\frac{\omega+\Omega}{2} t\right)
$$

For the small difference between frequencies $\omega$ and $\Omega$ the trembling effect is added to the harmonic vibration. The smaller the frequency difference is the higher the trembling period. 


\section{Examples}

Here three numerical examples are considered in an oscillator: 1. The excitation frequency is varied, 2. The excitation amplitude is varied and 3. The initial displacement is varied.Consider the vibrations of an oscillator with values $\alpha=\gamma=1$ and initial displacement $y_{0}=1$.

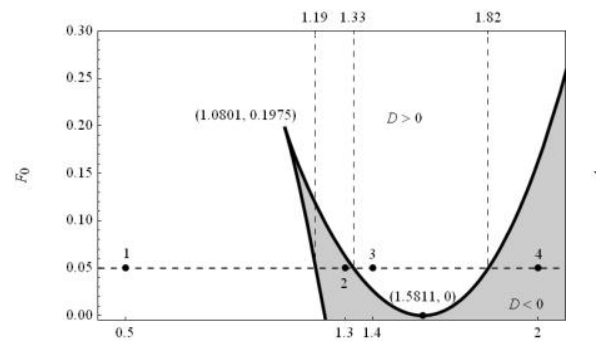

$\Omega$

Figure 3

$F_{0}-\Omega$ curve with characteristic points for $\alpha=\gamma=1$ initial displacement $y_{0}=1 \alpha=\gamma=1$

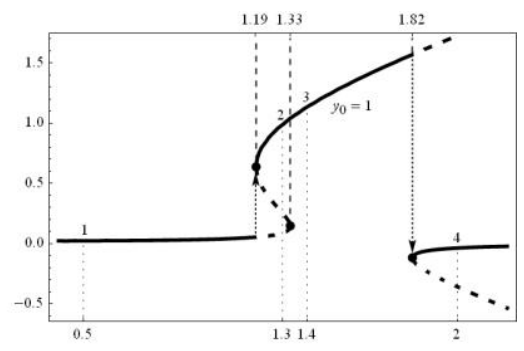

$\Omega$

Figure 4

$A-\Omega$ curve with characteristic points for and $F_{0}=0.05$ and initial displacement $y_{0}=1$

In Fig. 3 the $F_{0}-\Omega$ curve is plotted. The $F_{0}-\Omega$ curve separates the region where $D>0$ and the region where $D \leq 0$ (shaded area). Four $\left(\Omega, F_{0}\right)$ sets of excitation parameters are selected: point $1(0.5,0.05)$ for $D>0$, point $2(1.3,0.05)$ for $D<0$, point $3(1.4,0.05)$ for $D>0$, and $(2,0.05)$ for $D<0$. In Fig. 3 the characteristic points calculated in Table 1 for $y_{0}=1$ and also the peak values of $F_{0}-\Omega$ are given. In Fig. 4 the $A-\Omega$ curve for $F_{0}=0.05$ is plotted. The points $1-4$ are plotted and the numeric values of the characteristic points are signed. In Fig. 5 the $y$ - $t$ diagrams obtained analytically and numerically for the four mentioned parameter values are plotted.

From the Figures the following is evident:

For the parameter values $\alpha=\gamma=1$, initial displacement $y_{0}=1$, and excitation values $F_{0}=0.05$ and $\Omega=0.5$ (point 1 ) the mathematical model of the system is $\ddot{y}+y+y^{3}=0.05 \cos (0.5 t)$. According to the previous consideration the approximate analytical solution is $y=0.02291 \cos (0.5 t)+0.97709 \cos (1.3103 t)$. Point 1 is in the region $\Omega<\Omega_{1}=1.1931$, where only one solution for $A$ exists (see Fig. 4). 


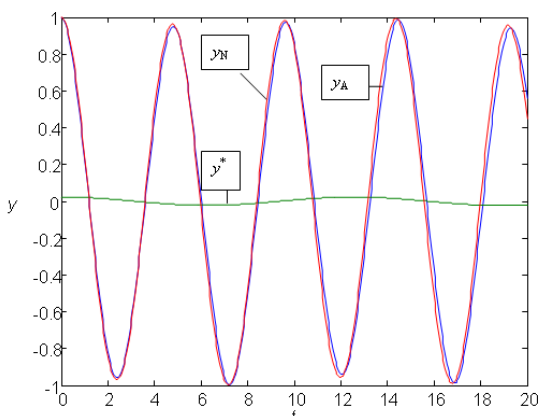

(a)

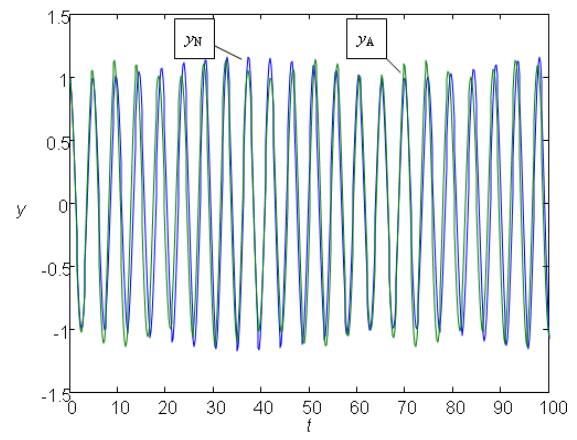

(c)

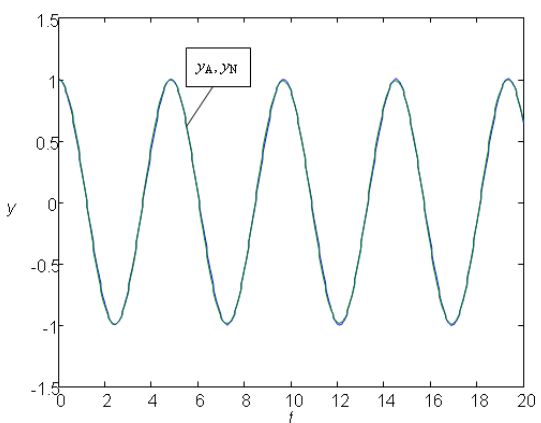

(b)

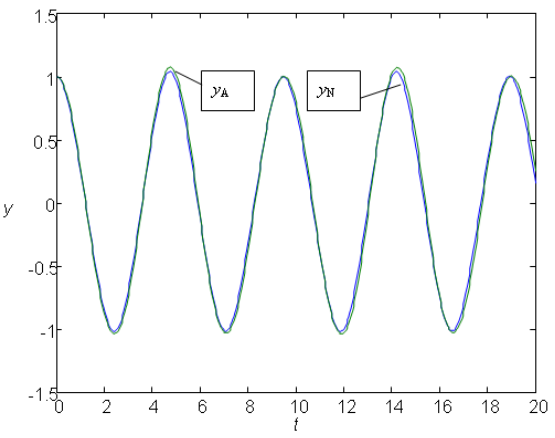

(d)

Figure 5

Time history diagrams obtained analytically $\left(y_{A}\right)$, numerically $\left(y_{N}\right)$ and the 'carrying curve' $y^{*}$ for $y_{0}=1$, $\alpha=\gamma=1, F_{0}=0.05$ and:a) $\Omega=0.5$, b) $\Omega=1.3$, c) $\Omega=1.4$ and d) $\Omega=2$

In Fig. 5a the analytical and numerical solution are plotted. The difference between the time history diagrams $y$ - $t$ is negligible. The vibration has two terms: the first is with amplitude $A<<y_{0}$ and with period $4 \pi$, and the second vibration term is with frequency $\omega<\omega_{0}$. In Fig. 5a it has been shown that the first term in the analytical solution can be treated as the 'carrying vibration' and the second term is the 'carried vibration' which is along the first one. The total vibration is with maximal amplitude $y_{0}$.

Differential equation of motion for $\Omega=1.3$ (point 2) is $\ddot{y}+y+y^{3}=0.05 \cos (1.3$ ). Point 2 is in the region $\Omega_{1}=1.1931<\Omega<\Omega_{2}=1.3295$, where three real solutions for $A$ exist (Fig. 3): $A_{1}=0.25058, A_{2}=0.089264, A_{3}=0.99349$. As for $\Omega_{1}=1.1931$ the jump phenomena for amplitude $A$ occurs, (see Fig. 4), the biggest value $A_{3}$ is valid for further analytical calculation. The approximate analytical solution is $y=0.9935 \cos (1.3 t)+0.0065 \cos (1.575 t)$. In Fig. $5 b$ the analytical solution is compared with numerically obtained one by solving the differential equation. As the amplitude $A \gg>B$, the term with excitation frequency $\Omega$ is dominant. For this 
case the term with excited frequency $\omega$ can be treated as a correction function. The approximate solution $y \approx 0.9935 \cos (1.3 t)$ is almost harmonic with amplitude close to $y_{0}$.

Point 3, with excitation frequency $\Omega=1.4$, is in the region $\Omega_{2}=1.3295<\Omega<\Omega_{3}=1.8191$ where only one real solution for $A$ exists (Fig. 3). The calculated value is $A=1.0717$ and is higher than the initial value of the amplitude $\left(y_{0}=1\right)$ while coefficient $B$ is negative $(B=-0.0717)$. For $\ddot{y}+y+y^{3}=0.05 \cos (1.4)$ the approximate solution is $y=1.0717 \cos (1.4 t)-0.717 \cos (1.6513 t)$. In Fig. 5c the analytical and numerical solutions $y-t$ are plotted. The obtained solutions are in good agreement. From the analytical solution it has been seen that the term with excitation frequency $\Omega=1.4$ is still the dominant one. Comparing the analytical solution by neglecting the second term as a small value, with the free vibration it has been concluded that the amplitude of vibration is higher and the period of vibration is shorter.

The excitation frequency $\Omega=2$ in point 4 is in the region $\Omega>\Omega_{3}=1.8191$ where three real solutions for $A$ (see Fig. 4) can be calculated $\left(A_{1}=-3.5994 \times 10^{-2}, A_{2}=-\right.$ $0.35752, A_{3}=1.7268$ ). For $\Omega_{3}=1.8191$ there is a jump in the value of $A$ and the smallest absolute value of $A$ corresponds to the real vibrating system. Thus, for $\Omega=2$ (point 4) the solution is $y=1.03599 \cos (1.3442 t)-0.03599 \cos (2 t)$. In Fig. $5 d$ the analytically obtained solution is compared with the numerical result, which is the solution of the differential equation $\ddot{y}+y+y^{3}=0.05 \cos (2 t)$. The second term in the approximate solution is negligible in comparison to the first term. Comparing the approximate solution $y \approx 1.03599 \cos (1.3442 t)$ with $y=1 \cos (1.322 t)$ for the free vibrations of the oscillator [15] described with $\ddot{y}+y+y^{3}=0$ it can be seen that the amplitude and frequency of vibration of the harmonically excited oscillator is smaller than for $\Omega=1.4$ and tends toward the properties of the free oscillator.

In Fig. 6 the time history diagram for $\alpha=\gamma=1, \Omega=1.3$, initial amplitude $y_{0}=1$ and excitation amplitude $F_{0}=0.25$ is plotted. There is only one real solution for $A$ (see Fig. 3) which gives the approximate analytical solution $y=1.0976 \cos (1.3 t)$ $0.0976 \cos (1.6776 t)$, which in comparison with the numerical one shows good agreement. Comparing Fig. 6 with Fig. 5b the influence of increase of the excitation amplitude on the vibration properties of the oscillator is visible. For a higher value of $F_{0}$ the trembling effect is evident, while for smaller $F_{0}$ the motion with approximately constant amplitude occurs.

In Fig. 7 the time history diagrams for an oscillator with $\alpha=\gamma=1, \Omega=1.5, F_{0}=0.05$ and various initial conditions $\left(y_{0}=0.5\right.$ and $\left.y_{0}=1\right)$ are plotted. It has been shown that the oscillating properties deeply depend on initial displacement. 


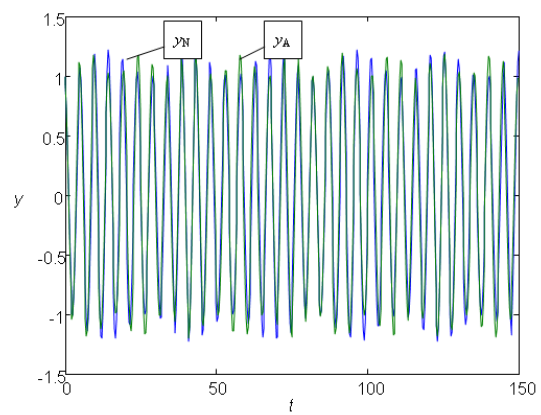

Figure 6

Analytical $y_{A}-t$ and numerical $y_{A}-t$ diagrams for $\Omega=1.3, \alpha=\gamma=1, F_{0}=0.25$ and $y_{0}=1$

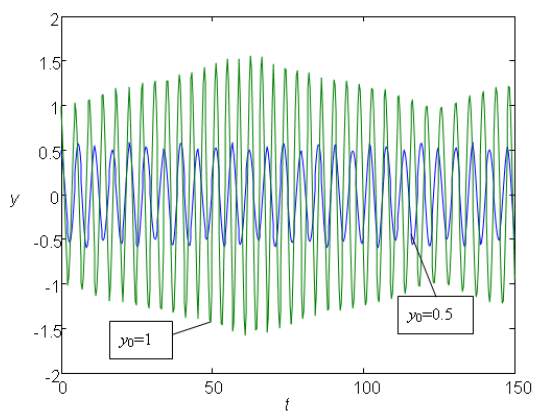

Figure 7

Time history diagrams for $\Omega=1.4, \alpha=\gamma=1, F_{0}=0.05$

and: $y_{0}=0.5$ and $y_{0}=1$

\section{Conclusions}

The following has been concluded:

1) The vibration of a non-linear undamped harmonically excited oscillator depends on initial conditions and their influence cannot be neglected independently on the value of the excitation parameters.

2) Upon analyzing the total vibration of the harmonically excited Duffing oscillator, it can be concluded that it contains two terms: first, a harmonic vibration with excitation frequency and second, a harmonic vibration with excited frequency. When varying the excitation frequency from zero to a higher values the vibration terms vary as follows:

a) For zero excitation frequency the first term is a constant value and the second term is a harmonic vibration. The sum of these two terms yields the vibration around the constant value and the maximal amplitude corresponds to initial displacement.

b) By increasing the excitation frequency the first term transforms into a harmonic function with a long vibration period: the period of vibration decreases and the amplitude of vibration decreases from the constant value by increasing the excitation frequency. For the second term, the period of vibration decreases and the amplitude increases when the excitation frequency increases. The total vibration represents the vibration of the second term, around the first one and the total amplitude is equal to the initial displacement.

c) By further increasing the excitation frequency, the relation between the frequencies of vibration of the first and the second term give the trembling effect, where the total vibration period decreases by increasing the excitation frequency and the amplitude remains equal to the value of the initial displacement.

d) For a further increase of excitation frequency, the second term becomes a dominant vibration with a longer period than that of the first term. The period of 
trembling increases and the difference between the maximal and minimal amplitude decreases.

e) By further increase of the excitation frequency, up to a certain value, when the amplitude of the first term is equal to initial displacement and of the second term is zero, the total vibration is a pure harmonic, with an amplitude equal to initial displacement and with that certain excitation frequency. For such excitation force, the vibration response is independent on the elastic properties of the oscillator.

f) For an even further increase of excitation frequency, the trembling effect appears, but with a positive sign: the period of the first term decreases and its amplitude is higher than the initial displacement while the frequency of the second term increases and the amplitude is smaller than initial displacement. In spite of that, the total amplitude of vibration is higher than initial displacement. By further increase of excitation frequency the trembling in vibration of the excited oscillator disappears and the vibration tends to a periodical with an amplitude equal to initial displacement and frequency which tends to the frequency of free vibration of the oscillator.

g) For significantly high excitation frequency, the excitation force has only a marginal influence on the vibration of the oscillator. Namely, the amplitude of vibration and the frequency of the excited oscillator do not depend on the parameters of excitation. The vibration is almost harmonic in nature, with an amplitude equal to initial displacement and with frequency of a free vibration. Based on this conclusion, it is recommended to use an excitation force with a high excitation frequency.

3) The initial displacement has an influence on the excitation domain which gives one, two or three steady-state vibrations. The excitation amplitude - excitation frequency curve is single-valued, if the initial displacement is zero and it separates the domains of one and three steady state motions equally. For arbitrary initial displacement, the excitation curve is multi-valued: for excitation parameters inside the region of this curve, three steady-state motions exist, while outside this region the motion only one steady state amplitude exists. The obtained region is larger for higher values of initial displacement. The higher the value of initial displacement the peak values move toward higher values of excitation frequency and excitation amplitude.

4) The number of peak amplitudes, which define the position of jump phenomena, depend on initial displacement. If the initial displacement is zero, only one characteristic peak value in the excited undamped Duffing oscillator exists.

\section{Acknowledgement}

This work was partially supported by the Secretariat for Science and Technological Development, Autonomous Province of Vojvodina (Proj. No 114451-2094/2011) and Ministry of Science of Serbia (Proj. No. ON 174028 and IT 41007). 


\section{References}

[1] Burton, T. D.: A Perturbaion Method for Certain Non-Linear Oscilltors, International Journal of Non-Linear Mechanics, 19 (1984) 397-407

[2] Cheng, Y. K., Chen, S. H. and Lau, S. L.: A Lindstedt-Poincare Method for Certain Strongly Non-Linear Oscillators, International Journal of NonLinear Mechanics, 26 (1991) 367-378

[3] Friswell, M. and Penny, J. E. T.: The Accuracy of Jump Frequencies in Series Solutions of the Response of a Duffing Oscillator, Journal of Sound and Vibration, 169 (1994) 261-269

[4] Worden, K.: On Jump Frequencies in the Response of the Duffing Oscillator, Journal of Sound and Vibration, 198 (1996) 522-525

[5] Qaisi, M. I.: Analytical Solution of the Forced Duffing's Oscillator, Journal of Sound and Vibration, 194 (1996) 513-520

[6] He, J-H.: Modified Lindstedt-Poincaré Methods for some Strongly NonLinear Oscillations Part I: Expansion of a Constant, International Journal of Non-Linear Mechanics, 37 (2002) 309-314

[7] Amore, P. and Aranda, A.: Improved Lindstedt-Poincaré Method for the Solution of Nonlinear Problems, Journal of Sound and Vibration, 283 (2005) 1115-1136

[8] Patil, N. S. and Mallik, A. K.: Experimental Investigation of the Response of a Harmonically Excited Hard Duffing Oscillator, Pramana - Journal of Physics, 68 (2007) 99-104

[9] Kovacic, I. and Brennan, M. J.: On the Use of Two Classical Series Expansion Methods to Determine the Vibration of Harmonically Excited Pure Cubic Oscillators, Physics Letters A, 372 (2008) 4028-4032

[10] Brennan, M. J., Kovacic, I., Carrella, A. and Waters, T. P.: On the Jump-Up and Jump-Down Frequencies of the Duffing Oscillator, Journal of Sound and Vibration, 318 (2008) 1250-1261

[11] Mester, Gy.: Intelligent Mobile Robot Motion Control in Unstructured Environments, Acta Polytechnica Hungarica, 7 (2010) 153-165

[12] — : The Duffing Equation: Nonlinear Oscillators and their Behaviour, Eds: Kovacic, I. and Brennan, M. J. (2011) Wiley

[13] Vahidi, A. R., Babolian, E. and Asadi Cordshooli, Gh.: Numerical Solutions of Duffing's Oscillator Problem, Indian Journal of Physics, 86 (2012) 311-315

[14] Cveticanin, L.: Forced Pure Nonlinear Symmetrical Oscillators, Mathematical and Computer Modelling, 55 (2012) 1580-1593

[15] Cveticanin, L.: The Approximate Solving Methods for the Cubic Duffing Equation Based on the Jacobi Elliptic Functions, International Journal of Nonlinear Sciences and Numerical Simulation, 10 (2009) 1491-1516 